\title{
FEMALE PSEUDOHERMAPHRODITISM
}

\section{Bulotta AL, Varetti C, Ferrara F, Giannotti G, Di Maggio G, Garzi A, Messina M}

\author{
Division of Pediatric Surgery, Department of Pediatrics, Obstetrics and Reproductive Medicine \\ University Of Siena
}

Introduction. $21 \alpha$-hydroxylase deficiency is the most frequent cause of virilization in patients with female karyotype due to exposure of a female fetus to excess of androgen. We report anatomical and cosmetic results of feminizing genital reconstruction of two related patients (second cousin) with XX karyotype born with urogenital sinus anomalies (UGS) and not treated at birth. Materials and Metods. Patient 1 is 6-years old with ambiguous genitalia graded as Prader V and never undergone therapy or surgery. Patient 2 is 10 -years old, graded as Prader IV and subjected to hormonal therapy and clitoral amputation at the age of 6. Mobilization of urogenital sinus, pull-through of vagina and tubulization of urethra was performed in both after placement of Foley chateters in vagina and bladder by cisto-vaginoscopy. Genitoplasty involved refashioning the tissues to create minora and majora labia and, after removal of corpora, partial clitorectomy was carried in patient 1 and clitoridal reconstruction in patient 2. Result. Vaginal introitus was positioned in the vestibule region below urethral meatus. Foley chateters was removed after two weeks in narcosis and the cosmetic and anatomic result was good. Conclusion. Goals of feminizing genitoplasty are to restore, soon as possible, anatomy achieving a more feminine appareance with a vagina for menstruation, to preserve reproductive capacity and to prevent urological sequelae but it's also important to contribute in a development of a more stable gender identity. This procedure in two stage, based on an accurate diagnosis, is good to create feminine genital appareance in children with female pseudohermaphroditism expecially if not treated at birth.

Keywords: Female Pseudohermaphroditism, Congenital Adrenal Hyperplasia (CAH), Urogenital Sinus (UGS), Vaginoplasty, Clitoroplasty, Labioplasty

\section{INTRODUCTION}

The female pseudohermaphroditism is an incomplete feminization or complete masculinization of patients with XX karyotype. The most common cause is $21-\alpha$ hydroxylase deficiency for congenital adrenal hyperplasia (CAH) with world incidence of 1:14,500. The degree of masculinization of the external genitalia is though to be related to the time and amount of androgen exposition in the fetus. The internal structures are normal (uterus, tubes, ovaries and upper vagina) since there isn't anti-Mullerian hormone, but it is possible a variable degree of external virilization. Prader's classification (1-5 grade) is usefull to describe the phenotype, while radiographic and endoscopic evaluation are indispensable and form the basis for surgical reconstruction. Both are performed to delineate the anatomy and should identify the length of the common sinus, the location of the confluence of the vagina, the distance from the vaginal confluence to the bladder neck, the presence of cervix and the normalcy of the bladderl.

Debate exists regarding the optimal timing of surgery, but anyway precocity of genital reconstruction or transformation is keystone of therapeutic procedure in these severe anomalies so to prevent discordance between genital sex and gender identity. The child who present tardily, requires a multidisciplinary approach (surgical, psychological, endocrinological) with the aim to prevent psychological or behavioural relapses2.

Furthermore, since the degree of virilization and level of the confluence of vagina with the urogenital sinus (UGS) is variable and unique to each girl, there are several options for surgical repair, which must be matched to the anatomy of the child to achieve a functional and cosmetic outcomel,3.

We report anatomical and cosmetic results of feminizing genital reconstruction of two related patients (second cousin) with XX karyotype born with urogenital sinus anomalies and not treated at birth.

\section{CASE REPORTS}

\section{Case 1}

I.F.S., a girl born at term by natural childbirth after a normal pregnancy. She presented a birth weight 3100 g (gravida 4 para 5), no electrolytes'alterations and ambiguous genitalia. Patient was raised as girl and she has never taken drugs including hormones. When she presented in our hospital at the age of six years, she characterized by ambiguous genitalia, graded as Prader

Correspondence to:

Prof. Mario Messina, Division of Pediatric Surgery, Dept of Pediatrics, Obstetrics and Reproductive Medicine.

University of Siena. Policlinico "Le Scotte" - Viale Bracci - 53100 - Siena - Italy

Telefono: +39577586501 - Fax: +39577586174

E-mail: messinam@unisi.it 
$\mathrm{V}$, with clitoromegaly, opening of the urogenital sinus on apex of the phallus, complete labioscrotal fusion, absence of palpable gonads bilaterally in inguinal canal and in labioscrotal folds, phallus' length $5 \mathrm{~cm}$ and presence hair in scrotal median raphe. Furthermore, to the clinical examination, it could be observed male muscles and absence of axillary hair. Perinatal history not revealed the maternal virilization or maternal medication used in pregnancy and there weren't prenatal karyotype and routine ultrasonography that might show presence of genital ambiguity. A family history revealed a cousin (case 2 ) affected by genitalia virilisation and also consanguinity between the parents. Laboratory investigation showed high levels of 17-OH-progesterone $(9.5 \mathrm{ng} / \mathrm{ml})$, androstenedione $(>10 \mathrm{ng} / \mathrm{ml})$, ACTH $(97.4 \mathrm{pg} / \mathrm{ml})$ and testosterone $(0.86$ $\mathrm{ng} / \mathrm{ml}$ ) but normal level of cortisol (136.5 ng/ml). There were normal $\mathrm{Na}+(139 \mathrm{mg} / \mathrm{dl})$ and $\mathrm{K}+(4.2$ $\mathrm{mEq} / \mathrm{l})$ levels. Bone age was 9.3 years ahead than chronological age (6.4 years). QF-PCR was carried and it revealed the female gender confirmed by the karyotype.

Ultrasound of abdomen and pelvis showed a hypoechoic formation behind bladder and absence of gonads. MRI was performed and it revealed a pyriform bladder, hydrometrocolpos and presence of high urogenital sinus (Fig.1). The precise location of the vaginal confluence in relation to the bladder neck and meatus was determined endoscopically using a catheter calibrated to the nearest centimetre as a guide. There was a male urethra and the distance from confluence to the urogenital sinus meatus was $6 \mathrm{~cm}$. Furthermore, laparoscopy was performed to assess the internal structures: uterus, tubes and ovaries were normal. To the light of these results 21- $\alpha$ hydroxylase partial deficiency with only virilization for congenital adrenal hyperplasia (CAH) was diagnosed and for normal level of cortisol any hormonal replacement was pro-

Fig.1 (right):

MRI image of pelvis in girl with $\mathrm{CAH}$; we see a pyriform bladder, hydrometrocolpos and the presence of high urogenital sinus with male urethra.

\section{Se:25 Im:45}

vided.

For surgical correction, two stages were needed: first vaginoplasty and second clitorolabioplasty. After placement of Foley chateters in vagina and bladder by cisto-vaginoscopy, prone approach allowed the midline incision and the opening of ventral side of sinus and the redundant tissue could be used to create a mucosalined vestibule. Finally vagina was mobilized towards perineum and posterior vaginal wall spatuled and anastomosed to perineum, while sinus was tubularized to create urethra (Fig.2). Foley chateters was removed after two weeks in narcosis and the cosmetic and anatomic result was satisfactory without disturbing of urethral sphincter. Clitoridolabioplasty was performed after one months. Clitoral reduction was done with preservation of the dorsal neurovascular bundle while the corporal bodies were excised from the insertion in the glans to the bifurcation of the corpora. The glans was sutured to the fascia underneath the symphysis pubis. Labioplasty was done by midline division of the preputial and phallic skin which was sutured to the lateral egdes of the opened UGS and to adjacent perineal skin. After surgery the girl performed vaginal dilatation with Hegar to avoid vaginal or introital stenosis.

\section{Case 2}

A.H., a girl born at term by natural childbirth after a

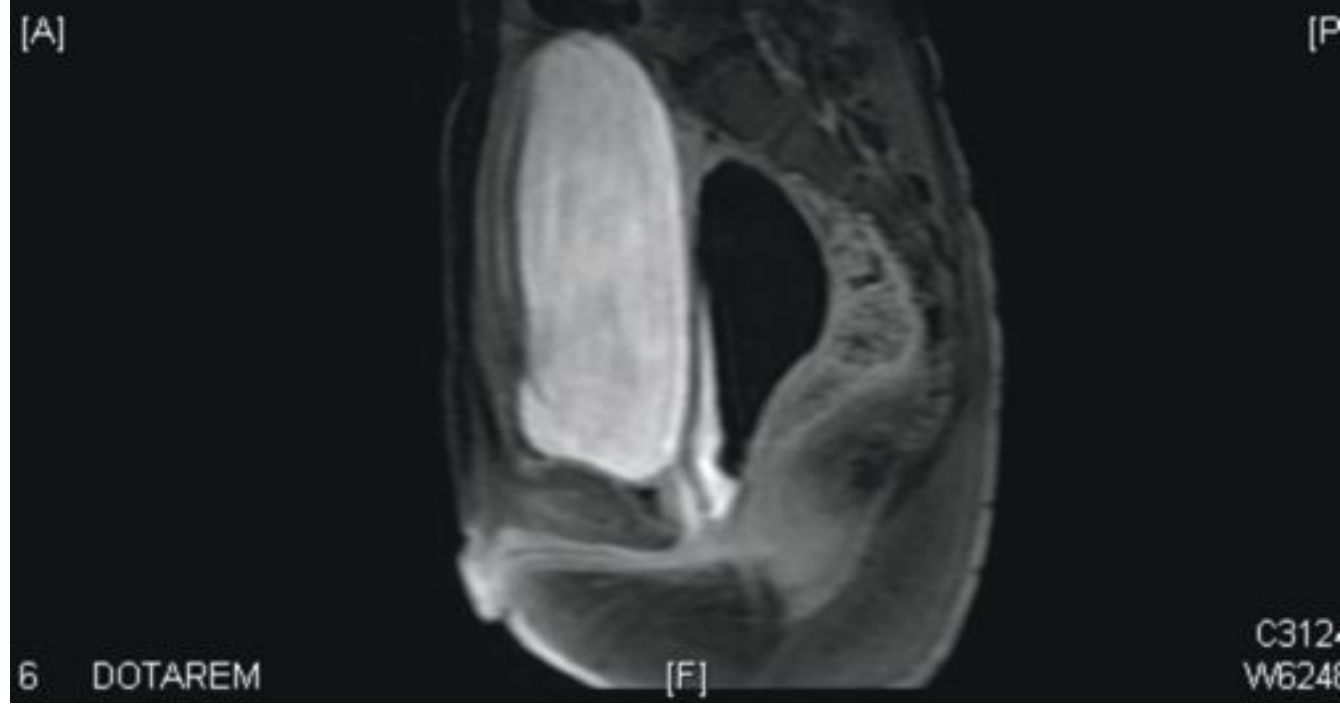


normal pregnancy. She presented a birth weight of 3500 g (gravida 1 para 3), no electrolytes'alterations and ambiguous genitalia. The child was raised female and for this reason at six years of age, clitoridal amputation and estrogen therapy was performed. The girl who is now ten years old presented to us at the clinical examination ambiguous genitalia, graded as Prader III, with posterior labioscrotal fusion and presence of urogenital sinus with a single opening in it, scar for clitoridal amputation, absence of palpable gonads bilaterally in inguinal canal and in labioscrotal folds, pubic hair, male muscles and axillary hair. Perinatal history not revealed exogenous virilization. A family history revealed consanguinity between the parents and kinship with patient before described. Laboratory investigation showed high levels of 17-OH-progesterone $(15.4 \mathrm{ng} / \mathrm{ml})$, androstenedione $(>10 \mathrm{ng} / \mathrm{ml})$, ACTH (602 pg/ml), testosterone $(3.72 \mathrm{ng} / \mathrm{ml})$ and cortisol $(59.8 \mathrm{ng} / \mathrm{ml})$ levels. There were normal $\mathrm{Na}+$ (136 $\mathrm{mg} / \mathrm{dl})$ and $\mathrm{K}+(4.2 \mathrm{mEq} / \mathrm{l})$ levels. No hypertension was documented. Bone age was 13 years ahead than chronological age (9.7 years). QF-PCR and karyotype were carried and revealed the female gender $46 \mathrm{XX}$. Ultrasound of abdomen and pelvis showed the presence of mass in left adrenal gland. MRI was performed and it revealed a normal bladder, uterus, vagina, ovaries and the presence of low urogenital sinus, left adrenal hyperplasia and normal corpora. Cistovaginoendoscopy was performed to evaluate the length of USG that was evaluated about $3 \mathrm{~cm}$. In this patient 21 $\alpha$ hydroxylase deficiency with only virilization was diagnosed and hormonal replacement with hydrocortisone and fludocortisone acetate was provided. Periodic laboratory reevalutations revealed a rapid normalization of androstenedione and a significant decrease in 17-OH- progesterone, testosterone and ACTH levels.

Surgical correction provided vaginoplasty, labioplasty and remotion of corpora. A complete reconstruction of clitoris wasn't possible because of previous excision of all clitoral tissue. After general anesthesia with hydrocortisone dose increase, the child was placed in the dorsal lithotomy position for endoscopy with insertion of Foley chateters in vagina and bladder. In prone position, a vaginal pull-through was performed after separation of anterior vaginal wall from the urinary tract. The UGS was closed to make the urethra and a posterior flap was used to rebuild the posterior vaginal wall. Foley chateter in vagina accidentally was removed in fifth day, while catheters in urethra was removed after two weeks postoperatively and vaginal dilatations were performed to prevent stenosis. In second stage, after one months, a inverted-Y- incision was performed on vaginal ostium to allow a major calibre with resorbable Vycril 5.0. Besides removal of corpora, labioplasty by residual tissue of the scrotum and aesthetical partial reconstruction of denervated clitoral was performed. A Penrose's drain was positioned near residues of corpora.

\section{DISCUSSION}

2006 Consensus Statement established a new nomenclature for ambiguous genitalia providing the term Disorders of Sex Development and includes congenital conditions in which development of chromosomal, gonadal or anatomical sex is atypical4. We prefer to use the old classification and hence term of female pseudohermaphroditism to give better phenotypic framework of the disease to the readers.

Management of intersex conditions frequently poses a challenge to pediatric endocrinologist, pediatric surgeons and psychologist. Indeed it isn't simple to make a decision that will influence the infant's future especially when the diagnosis is made late.

Therefore the decision is based on many consideration like the gender identity (referring to one's sense of belonging to the male or female sex category), capacity for normal sexual function and potential fertility, genotype, surgical options, endocrine function and testosterone imprinting. In fact clinical studies in girls with $\mathrm{CAH}$ have confirmed widely the testosterone imprinting on the fetal brain 5,6.

We reported two cases of classical CAH with only virilisation in which the genital ambiguity was not a previous reason of concern. These children were intrauterine exposed to excessive quantities of androgens, and they had normal internal genitalia but external ambiguous genitalia. In fact ultrasound and MRI confirmed presence of uterus, ovaries and tubes and provided an accurate description of UGS's anatomy. Only after having a clear diagnostic framework through hormonal, radiographic and endoscopical results, we could proceed with the surgical strategy. Many different surgical techniques have been described for the treatment of severely virilezed patients with a long USG $(>2,5 \mathrm{~cm})$ secondary to intersex disorders assigned to the female gender. Apart from the benefits and disadvantages of each one of these surgical procedures, it is quite clear that the management of these patients must take into account which is the most suitable surgical technique to give the urinary tract and genital apparatus as normal an anatomical state as possible, while also providing physiologic functions 1,7,8. Three are the basic components to feminizing genitoplasty: vaginoplasty, clitoroplasty and labioplasty. Debate still exists regarding when each of these components should be performed. We prefer the approach in two stage (first vaginoplasty and second labioplasty and clitoroplasty) because it allows to patients to address better and to adapt to the trasformation when diagnosis is late and also the tissues can to overcome better the surgical stress.

The present genital reconstruction includes separation of the vagina from urethra, vaginal exteriorization, reduction clitoridoplasty in case 1 and whenever possible partial reconstruction as in case 2 , and creation of the labia majora and minora. It's essential to perform cisto-vaginoendoscopy at the time of the surgery to confirm the position of the urethrovaginal confluence and to position Foley's chateters in vagina and bladder. Besides prone position provided a good exposition of 
perineum and a easy access to the USG. Our surgical choice provided a sagittal posterior access and vaginoplasty with posterior flap by resorbable Vycril 5.0.. This allowed us to perform a tension-free in epithelial to epithelial anastomosis between perineum and vagina and also to reduce possibility of vaginal stricture7. Clitoroplasty and ablation of corpora was performed to avoid painful erections and a poor aesthetic result with conservation of corporal, 9.

After two weeks Foley's catheters were removed in narcosis to help healing of tissues and to allow the urethra and vaginal ostium to take a exact location in the new vestibule and also vaginal dilatations were performed to promote a suitable calibre.

In our patients vaginal strictures or glans necrosisdid not occurr. Besides cosmetic and anatomic result are good for size and position of the glands, location of the urethral and vaginal orifices in the vestibular region and presence of proportionate and feminine appearance of labial structures.

\section{Conclusions}

The management of patients with ambiguous genitalia requires an important effort by different figures order not only to provide the specific diagnosis but also a gender assignmen . Goals of feminizing genitoplasty are to restore, soon as possible, anatomy achieving a more feminine appareance with a vagina for menstruation, to preserve reproductive capacity and to prevent urological sequelae but it's also important to contribute in a development of a more stable gender identity5,10,11. This procedure in two stage, based on an accurate diagnosis, is good to create feminine genital appareance in children with female pseudohermaphroditism expecially if not treated at birth.

\section{REFERENCES}

1. Domini R., Ceccarelli PL., Domenichelli V., Centonze N. Genitali ambigui.
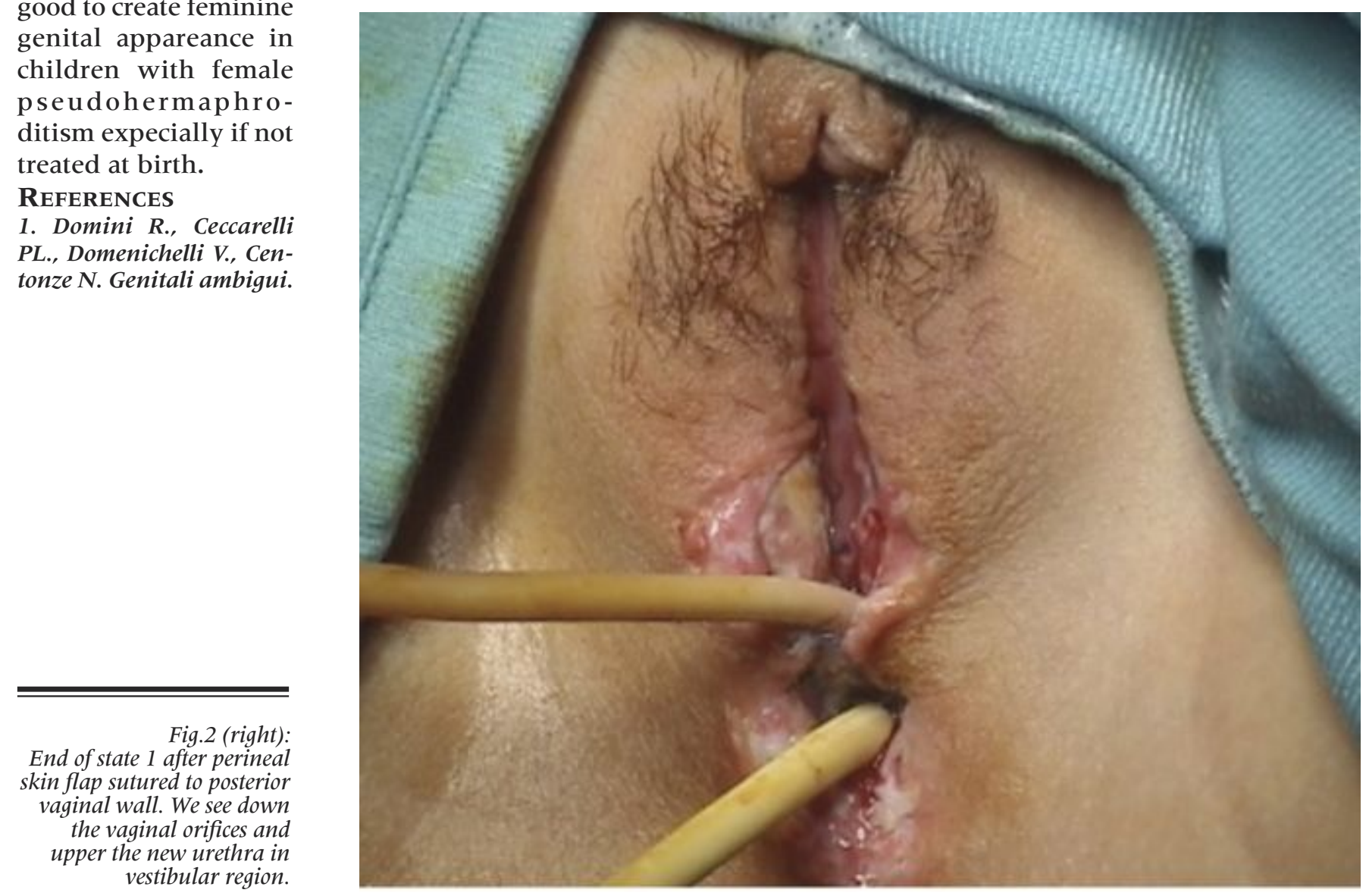

In: Domini, De Castro (eds): Chirurgia delle malformazioni urinarie e genitali, Padova, 1990, pp. 541-579

2. Intersex Society of North America Committee. Clinical Guidelines for the management of Disorders of Sex Development in Childhood. 2006

3. Jeffrey A. Leslie, Mark Patrick Cain, Richard Carlos Rink. Feminizing genital reconstruction in congenital adrenal hyperplasia. Ind J of Urol, 2009: 17-26

4. Lee PA, Houk CP, Ahmed SF, et al. Consensus statement on management intersex disorders. International Consensus Conference on Intersex. Pediatrics 2006; 118:e488-500

5. American Academy of Pediatrics. Evalutation of the newborn with developmental anomalies of the external genitalia. Pediatrics 2000; 106: 138-142

6. Hines M, Kaufman FR. Androgen and the development of human

sex-typical behavior: rough-and-tumble play and sex of preferred playmates in children with congenital adrenal hyperplasia (CAH). Child Dev. 1994;65:1042-1053

7. Miguel Podesta, Javier Urcullo. Perineal mobilization of the common urogenital sinus for surgical correction of high urethrovaginal confluence in patients with intersex disorders. $J$ Pediatr Urol. 2008;4:352-358

8. Rink CR, Pope JC, Kroop BP, Smith Jr, Keating MA, Adams MC. Reconstruction of the high urogenital sinus: early perineal prone approach without division of the rectum. J Urol 1997;158:1293-7

9. Kogan SJ, Smey P, Levitt SB. Subtunical total reduction clitoroplasty: a safe method of existing techniques. J Urol 1983; 130:746-748

10. Lerman Se, McAleer IM, Kaplan GW. Sex assignment in cases of ambiguous genitalia and its outcome. Pediatrics 2000;106:138-42

11. Eroglu E, tekant G, Gundogdu G, Emir H, Ercan O, Soylet $Y$, Danismend $n$. Feminizing surgical management of intersex patients. Pediatr Surg Int 2004; 20:543-547 